\title{
Area Under Curve
}

National Cancer Institute

\section{Source}

National Cancer Institute. Area Under Curve. NCI Thesaurus. Code C64774.

The area between the $x$-axis and the curve given by the integrand. It is equal to the definite integral of a function. In the field of pharmacokinetics, the area under the curve (AUC) is the area under the curve in a plot of concentration of a drug in plasma against time. AUC is usually given for the time interval zero to infinity, and other time intervals are indicated. 